grees of freedom, Fig. 1. Dynamic supersymmetries would now lead to energy formulae describing simultaneously sets of nuclei (supermultiplets), comprising both even-even and even-odd nuclei. The first experimental evidence of supersymmetry in nuclei was found in the spectra of the osmium-iridium nuclei, (Fig. 3), and is based on a classification in terms of the supergroup $U(6 / 4)$. Since then, several other examples have been found in the same and in other regions, corresponding to other supergroups $\mathrm{U}(6 / \mathrm{m})$. The search for even more examples is, at present, a very active area of research in Europe and throughout the world.

A general feature of the experimental examples found so far is that supersymmetry in nuclei appears to be more broken than normal symmetry. This is not an unexpected result since supersymmetry implies more stringent conditions on the interactions than norma symmetry. For example, it implies particular relations between the bosonboson, fermion-fermion and boson-fermion interactions. Nonetheless, supersymmetry appears still to be a useful tool in the study of spectra of odd-even nuclei. The full power of this tool has not yet been exploited.

\section{Conclusions}

Experimental examples of dynamic symmetries and supersymmetries have been found in the spectra of complex nuclei. These symmetries and supersymmetries have provided an elegant and concise classification of many nuclear spectra. In addition, they have given important clues for constructing a microscopic theory of collective states in nuclei, a subject not discussed here. Indeed, by studying this microscopic theory, one has been able to understand why a given nucleus can be described by a particular dynamic symmetry or supersymmetry.

The implications of the experimental discovery of supersymmetry in nuclei to other fields of physics are not yet clear. Supersymmetries observed in nuclei are of a type different from those mostly sought in elementary particle physics, since the bosons of the interacting boson model are actually correlated pairs of fermions (Cooper pairs) rather than fundamental objects. On the other hand, it may be that experimentally realizable supersymmetries are only of the type encountered in nuclear physics, i.e. where the bosons are composite rather than fundamental particles. In this connection, particularly interesting is some recent work of Y. Nambu (Chicago) who has discussed the analogy between the supersymmetry observed in nuclei and the motion of electrons around a vortex in a type II superconductor. Both problems arise in conjunction with spontaneous symmetry breaking of a nontrivial topological nature.

Be as it may, the occurrence of dynamic symmetries and supersymmetries in nuclei, has stimulated renewed interest in nuclear structure studies and led to major new advances in this field.

\section{REFERENCES}

1. Arima A. and lachello F., Phys. Rev. Lett. 35 (1974) 1069; Ann. Rev. Nucl. Part. Sci. 31 (1981) 75.

2. Arima A., Otsuka T., lachello F. and Talmi I., Phys. Lett. 66B (1977) 205.

3. Volkov V. D. and Akulov V. P., Phys. Lett. 46B (1973) 109.

4. Wess J. and Zumino B., Nucl. Phys. B70 (1974) 39.

5. lachello F., Phys. Rev. Lett. 44 (1980) 772; Balantekin A. B., Bars I. and lachello F. Phys. Rev. Lett. 47 (1981) 19.

\title{
Heavy-Ion Reactions
}

\author{
H. Doubre, Caen \\ (Grand Accélérateur National d'lons Lourds - GANIL)
}

Up to the mid-sixties, our knowledge of nuclear properties was confined to the individual behaviour of nucleons inside the mean field which is responsible for nuclear stability. However, the evidence for rather large deformations in several regions of the periodic table, and extensive studies of fission made it clear that many nuclear excitations exhibit a strongly collective aspect. Any nuclear model has to account for these collective features, which should ultimately be obtained from the nucleon-nucleon interaction only. At the extreme, nuclear theory introduced the concept of "nuclear matter" where any finite-size effect was neglected.

Heavy-ion collisions offer the opportunity of making large pieces of nuclear matter interact, and observing a host of extremely different collective phenomena. Each of them is a challenge to our

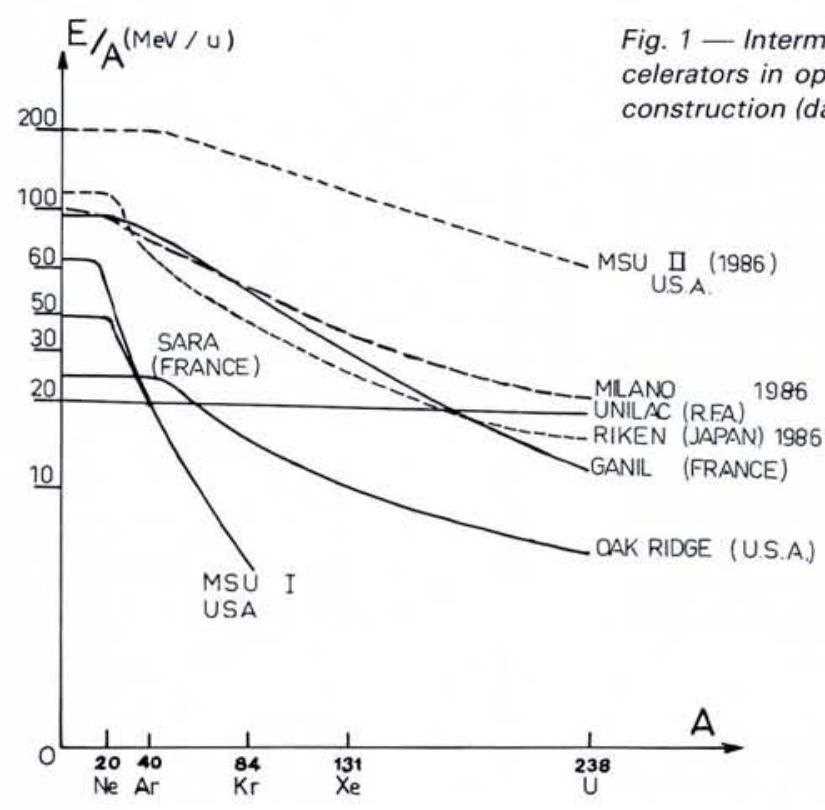

theories of nuclear models and reactions. The extreme variety of the experimental situations makes heavy-ion reaction studies a fascinating and rich field of investigation.

The acceleration of heavy-ions was made possible during the sixties, by a technical breakthrough in ion sources. To begin with, beams of carbon, nitrogen and oxygen ions were accelerated, but recently, uranium beams have been produced with energies ranging from 10 $\mathrm{MeV}$ to $0.96 \mathrm{GeV}$ per nucleon (u). The available energy range (in $\mathrm{MeV} / \mathrm{u}$ ) and the spectrum of ions to be accelerated are now used to characterize the machines in use and under construction Fig. 1.

Probably, the main interest initially found in heavy-ion collisions relied upon kinematical arguments: by an appropriate choice of the target-projectile pair

Fig. 1 - Intermediate-energy heavy ion accelerators in operation (full lines) or under construction (dashed) 


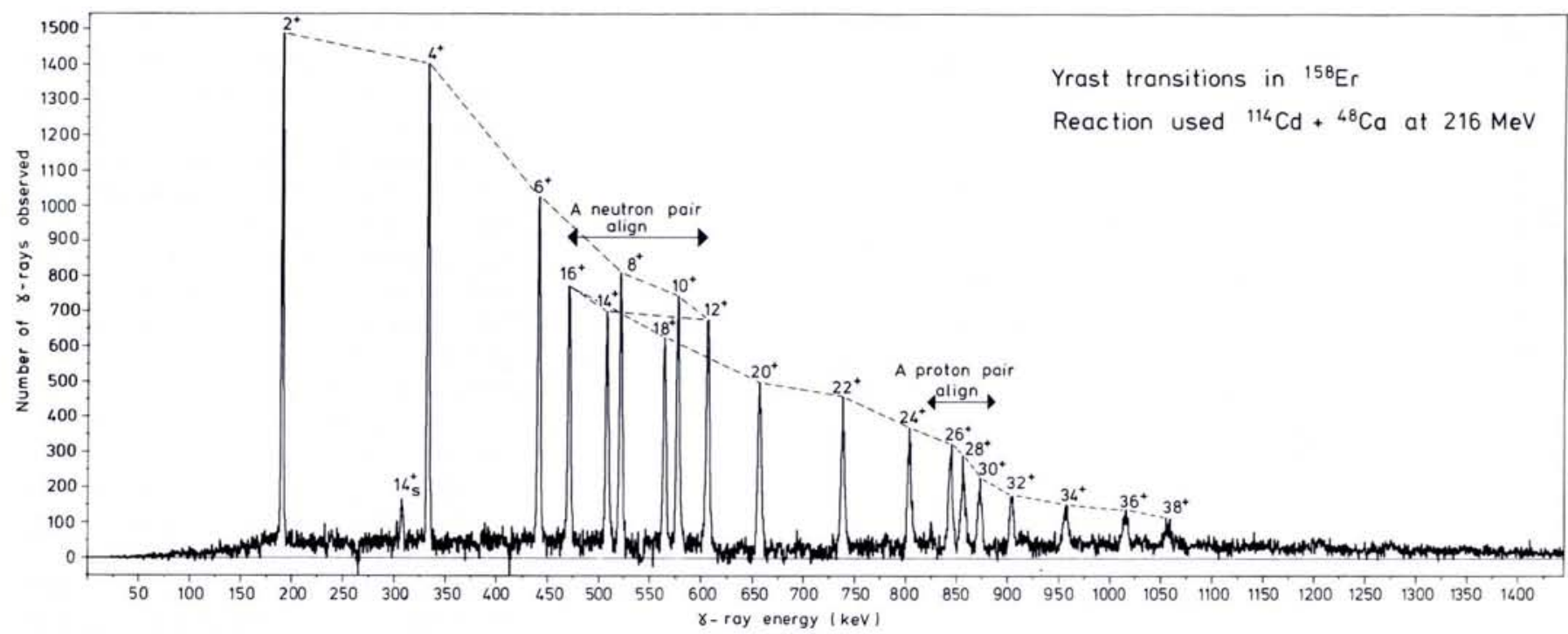

Fig. 2 - High-spin states in ${ }^{158} \mathrm{Er}$ from the reaction ${ }^{114} \mathrm{Cd}\left({ }^{48} \mathrm{Ca}, 4 n\right){ }^{158} \mathrm{Er}$ (courtesy J.F. Sharpey-Schafer).

and of the incoming energy, it is readily possible to choose the excitation energy, the linear momentum, the maximum angular momentum, etc. imparted to the target nucleus. Fusion reactions especially have taken advantage of this opportunity since a compound nucleus can be prepared with predetermined conditions, and one can select in advance the de-excitation path to be observed. With current 1) experimental techniques, electromagnetic transitions from excited states with spin higher than $40 \mathrm{~h}$ have been observed; in the rare-earth region, nuclei are predicted to sustain angular momenta larger than $90 \mathrm{~h}$ !

A fascinating field to study is the equilibrium shape of rotating nuclear matter ${ }^{2}$ ), the balance between the collective features of the motion and the influence of valence nucleons (which themselves bear a large intrinsic angular momentum in the same mass region), the influence of the Coriolis force on the pairing force, rotational band crossings, abrupt shape transitions due to the above phenomena ${ }^{3}$ ). Fig. 2 shows these high-spin studies, with the very favourable reaction ${ }^{48} \mathrm{Ca}+{ }^{114} \mathrm{Cd} \rightarrow 4 n$ $+{ }^{158} \mathrm{Er}$.
The fusion process, besides giving access to new and fascinating states of familiar nuclei, can be used for producing new nuclear species. It is wellknown that the extrapolation of nuclear theory (notably that dealing with shell effects) has led to the prediction that stable (or at least relatively stable) superheavy nuclei, with atomic number close to 116 can exist. The search for superheavy nuclei has not been successful yet, but recently, a careful analysis of the necessary conditions to make two nuclei fuse, as well as the use of very intense heavy-ion beams, has made it possible to synthesise ${ }^{4}$ ) the heaviest elements to date, with atomic numbers 107 and 109 .

During the systematic experimental study of the static and dynamic criteria for heavy-ion fusion, a new and very important class of reactions was discovered: the deeply inelastic collisions ${ }^{5}$ ). In these collisions, a large amount of the incident energy is "dissipated", that is, transformed into excitation energy of the participant nuclei, although the process remains binary, with a stochastic and limited exchange of nucleons. In view of the weak evidence for shell ef- fects at $Z=116$, the overwhelming probability of deeply inelastic collisions for very heavy ions, and the large excitation that a superheavy element would be formed with, its synthesis appears now as a formidable challenge indeed.

Even if this goal cannot be reached, the production of lighter, but very exotic nuclei (i.e. very far from the stability valley, an example could be ${ }^{70} \mathrm{Ca}$ ) appears possible via the fragmentation process. This process is highly favoured at the high energy (greater than 20 $\mathrm{MeV} / \mathrm{u}$ ) heavy-ion accelerators which are now built. It can be connected to the above mechanisms in the following way. At low energies, the mean free path of nucleons inside the nuclear matter largely exceeds the size of the nucleus (a fact upon which the independent-particle, shell-model of the nucleus is based). As the incident energy increases (beyond $20-30 \mathrm{MeV} / \mathrm{u}$ ) theory predicts that the mean free path shrinks and becomes comparable to the internucleon distance. Thus, at low energies, a nucleon only interacts with the walls of the mean field which contains all the nucleons of the studied system (this picture can also explain the dissipation ef-

\section{SENIOR PHYSICISTS}

Senior physicists who have retired from remunerative employment are reminded that they are entitled to apply for a $\mathbf{5 0 \%}$ reduction in their membership fee as an EPS Individual Ordinary Member.

Application should be made in writing to the Secretariat of the EPS in Geneva.
Further information: International Advanced Study Institute c/o Mrs. R.-M Vandekerkhof, Department of Physics, University of Antwerpen, Universiteitsplein 1, B - 2610 Wilrijk (Antwerpen, Belgium) 


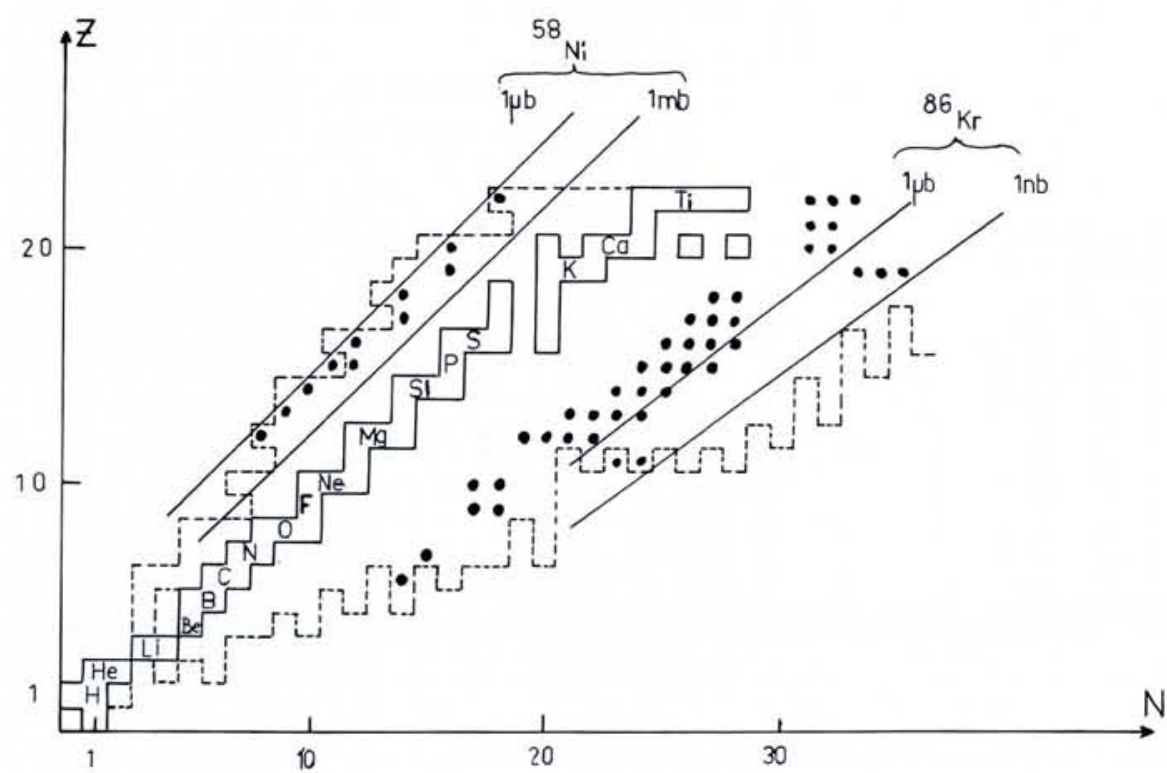

Fig. 3 - Search for exotic light nuclei. Dotted squares: predicted particle-stable nuclei. Dots: exotic nuclei from fragmentation reactions. The lines are predicted iso-cross-sections curves for fragmentation reactions with the indicated projectiles, such as could be expected from GANIL.

fects observed with deeply inelastic reactions, and is analogous to the behaviour of a rarefied Knudsen gas). At higher energies, the usual hydro-dynamical concepts of two-body interaction and viscosity become valid, and the interaction between two high-energy heavy ions is schematically depicted as an abrasion phenomenon and is called fragmentation.

The fast breakup has been shown to give rise rather easily to exotic nuclei as can be seen in Fig. 3 which depicts that part of the periodic chart where exotic nuclei have been produced with quite large cross-sections using this process. Recent measurements at GANIL have demonstrated that this process will largely dominate at energies between 10 and $100 \mathrm{MeV} / \mathrm{u}$.

We have seen that heavy-ion reaction studies lead not only to interesting physical situations for a nuclear physicist, but raise also many questions which are strongly connected with other fields of physics. For instance, the case for the existence of superheavy elements is concerned with the stability of a macroscopic system, on which quantal corrections are superimposed. Deeply inelastic reactions are considered as dissipative and diffusion processes, wherein the time scales for the propagation of different perturbations can be compared. These questions have all been largely studied by statistical mechanics; here they address themselves to systems with (only) several hundred degrees of freedom. We have also shown with the new accelerators (i.e. GANIL in France,
NSC at Michigan State University, RIKEN in Japan...) that the transition between cooperative aspects of the dynamics (observed at low energy) and the extreme nucleon-nucleon picture at high-energy needs to be studied in a detailed way.

The thermodynamical properties of nuclear matter are connected with the behaviour of supernovae, neutron stars, and the whole of stellar history. At the beginning of the seventies, the Lawrence Berkeley Laboratory took a lead in creating dramatically new physical situations: first to compress and thermally excite nuclear matter in order to explore its so-called equation of state (that is, the surface $\varepsilon \equiv \varepsilon(\rho, T)$ where $\rho$ and $\varepsilon$ are the baryonic mass and energy densities and $T$ the temperature), second to induce in the medium some predicted phase transitions: for instance pion condensation or transformation of nuclear matter into quark matter. Measurements with pions are extremely useful here, since pions are present from the first stages of the collision, and it can be inferred from theory that they give clues back to the history of the reaction. Fig. 4 shows how from pion data it has been possible to extract directly ${ }^{6}$ ) (in a model-dependent way) the curve $\varepsilon \equiv$ $\varepsilon(\rho)$ at $T=0$, and compares these results with an extrapolation of the ground-state properties.

The field of heavy-ion induced reactions continues to be extremely active. The first experiments led to results which are relevant to many domains of physics. The search for exotic nuclei, that is, pieces of nuclear matter subject to some distortions away from the main stability valley was at the origin of an intense and systematic study of interactions between heavy ions, and this study has proved to be extremely fruitful. At nearly the same time, the study of relativistic heavy-ion collisions, with the ambitious goals of reproducing in the laboratory some of the important stellar processes and looking at the completely unknown thermodynamics of nuclear matter, has led to a completely different picture of collisions.

As to current facilities (Fig.1), the Unilac in Darmstadt (FRG) was recently upgraded to $20 \mathrm{MeV} / \mathrm{u}$, and is deeply involved in the study of the onset of fragmentation. The SARA facility in Grenoble (France) produces "light" (up to Ar) heavy-ion beams up to $30 \mathrm{MeV} / \mathrm{u}$. Phase I, at Michigan State University (USA) is rated similarly. The only accelerator with the full spectrum of ions and energies up to $100 \mathrm{MeV} / \mathrm{u}$ is currently the "Grand Accélérateur National d'lons Lourds" (GANIL) in Caen (France). Other accelerators are actively being built in Japan and Italy, and at Michigan State University, Phase II is now programmed.

\section{REFERENCES}

1. Twin P., Proceedings of the International Conference on Nuclear Physics, Florence (1983), (Editrice Compositori, Bologna).

2. Cohen, Plasil F. and Swiatecki W., Ann. Phys. (N.Y.) 82 (1974) 557.

3. Diamond R.M. and Stephens F.S., Ann. Rev. Nucl. Part. Sci 30 (1980) 85.

4. Armbruster P., Europhysics News 14 (1983) 8/9.

5. Lefort M. and Ngo C., Ann. Phys. (Paris) 3 (1978) 5.

6. Stock R., G.S.I. Nachrichten 10-82.

Fig. 4 - Compressional energy of nuclear matter as a function of its density. The upper curve corresponds to a compressibility constant $K=250 \mathrm{MeV}$, the lower to the "standard" $\mathrm{K}=200 \mathrm{MeV}$. From Ref. 6 .

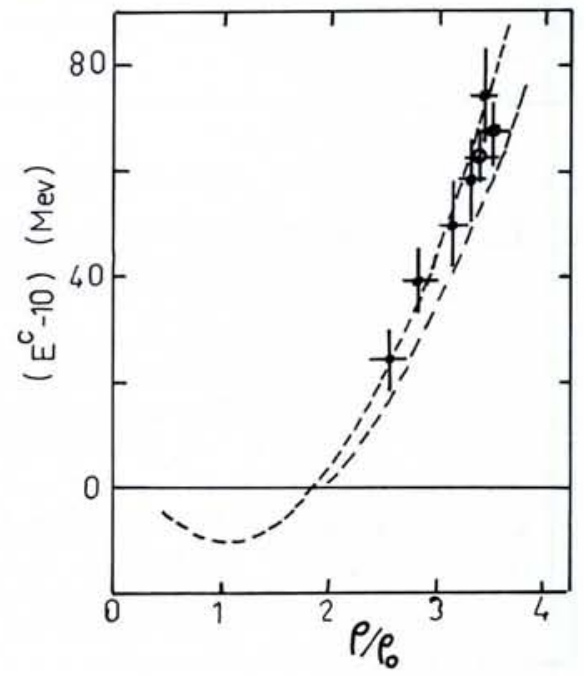

